\title{
Engineering of an E. coli outer membrane protein FhuA with increased channel diameter
}

\author{
Manuel Krewinkel, Tamara Dworeck and Marco Fioroni ${ }^{*}$
}

\begin{abstract}
Background: Channel proteins like FhuA can be an alternative to artificial chemically synthesized nanopores. To reach such goals, channel proteins must be flexible enough to be modified in their geometry, i.e. length and diameter. As continuation of a previous study in which we addressed the lengthening of the channel, here we report the increasing of the channel diameter by genetic engineering.

Results: The FhuA $\triangle 1-159$ diameter increase has been obtained by doubling the amino acid sequence of the first two N-terminal $\beta$-strands, resulting in variant FhuA $\Delta 1-159$ Exp. The total number of $\beta$-strands increased from 22 to 24 and the channel surface area is expected to increase by $\sim 16 \%$. The secondary structure analysis by circular dichroism (CD) spectroscopy shows a high $\beta$-sheet content, suggesting the correct folding of FhuA $\triangle 1-159$ Exp. To further prove the FhuA $\triangle 1-159$ Exp channel functionality, kinetic measurement using the HRP-TMB assay (HRP $=$ Horse Radish Peroxidase, $\mathrm{TMB}=3,3^{\prime}, 5,5^{\prime}$-tetramethylbenzidine) were conducted. The results indicated a $17 \%$ faster diffusion kinetic for FhuA $\triangle 1-159$ Exp as compared to FhuA $\triangle 1-159$, well correlated to the expected channel surface area increase of $\sim 16 \%$.

Conclusion: In this study using a simple "semi rational" approach the FhuA $\triangle 1-159$ diameter was enlarged. By combining the actual results with the previous ones on the FhuA $\Delta 1-159$ lengthening a new set of synthetic nanochannels with desired lengths and diameters can be produced, broadening the FhuA $\triangle 1-159$ applications. As large scale protein production is possible our approach can give a contribution to nanochannel industrial applications.
\end{abstract}

Keywords: Channel proteins, FhuA, liposomes, protein engineering, HRP, TMB-Assay, nanocontainers

\section{Background}

Integral outer membrane proteins of gram negative bacteria use amphiphatic $\beta$-sheets to traverse lipid membranes. $\beta$-barrel proteins consisting of $8,12,14,10,18$ and 22 strands are known. All members of the above mentioned family are cylindrical, closed barrels with an even number of transmembrane $\beta$-strands that are connected in a $\beta$-meander topology with alternating tight turns and longer connecting loops [1]. The $\beta$-strand contribution to the overall secondary structure of these proteins is usually high $(\sim 60 \%)$ [1-5]. The respective membranes are spanned by $\beta$-strands of 9-11 residues. The smallest known barrel (i.e. OmpA) contains 8 transmembrane strands; due to packing constraints in the

\footnotetext{
* Correspondence: m.fioroni@biotec.rwth-aachen.de Department of Biotechnology (Biology VI), RWTH Aachen University, Worringerweg 1, 52074 Aachen, Germany
}

barrel interior, this might mark the lower possible size limit [2]. The largest known $\beta$-barrel proteins contain 22 strands (i.e. TonB dependent importers). However there is some evidence for the existence of even larger $\beta$-barrels [3]. In general the hydrophobic and membrane-interacting surface of $\beta$-barrel proteins is cryptically encoded in their primary sequence [4].

Apart from their biological importance, one application of bacterial membrane proteins with $\beta$-barrel structure is the channel functionalization of lipid or block copolymer based membranes. So far the bacterial nucleoside transporter Tsx, which is one of the smaller $\beta$-barrel proteins with 12 antiparallel strands [5], the $E$. coli outer membrane protein $\mathrm{F}(\mathrm{OmpF})$, with 16 antiparallel $\beta$-strands [6], the E. coli mechanosensitive channel protein MscL and one of the largest $\beta$-barrel proteins, the $E$. coli Ferric hydroxamate protein uptake component A (FhuA) have been successfully inserted into lipid 
or polymer based vesicles [7-9]. Especially the FhuA proved to be useful, due to its wide channel diameter and robustness against for instance tryptic digestion [10].

The $E$. coli outer membrane protein FhuA is one of the largest known $\beta$-barrel proteins (714 amino acids, elliptical cross section $39^{*} 46 \AA$ ), consisting of 22 antiparallel $\beta$ strands connected by short periplasmatic turns and flexible external loops. The protein channel is closed by a cork domain (amino acids 5-159). Several crystal structures of the FhuA wild type have been resolved $[11,12]$. The number of amino acids spanning the outer membrane is 9 to 10 for each $\beta$-strand [12]. For biotechnological applications one FhuA variant has been engineered in which the cork domain has been removed (FhuA $\Delta 1-159$, i.e. deletion of amino acids 1 - 159), resulting in a passive mass transfer channel [13]. The FhuA $\Delta 1-159$ variant has been inserted as a nanochannel triggered by chemical external stimuli into PMOXA-PDMS-PMOXA [PMOXA - poly(2-methyl-2-oxazoline); PDMS - poly(dimethylsiloxane)] triblock copolymer membranes [14] or liposomes respectively [15]. Very recently FhuA $\Delta 1-159$ was specifically altered to insert it into thick membranes formed by cheap triblock copolymer $\mathrm{PIB}_{1000}-\mathrm{PEG}_{6000^{-}}$ $\mathrm{PIB}_{1000}(\mathrm{PIB}=$ poly-isobutylene, $\mathrm{PEG}=$ poly-ethylen-gly$\mathrm{col})$. For this purpose the protein hydrophobic region was elongated from 3 to $4 \mathrm{~nm}$ by "copy-pasting" the last five amino acids of each $\beta$-sheet on the periplasmatic side of the barrel [16].

Previous findings show that FhuA and its variants are applicable as nanochannels in liposome or polymersome systems. Furthermore engineered FhuA variants can be an alternative to nanochannels based on artificial $\beta$-barrel structures able to insert into the lipid bilayer [17-19]. These artificial and self-assembling $\beta$-barrels are based on rigid rod molecules - extremely rigid, synthetic rodshaped molecules with great potential in material sciences - combined with short peptide strands. Artificial $\beta$-barrels can in principle be tailored in size and functional groups $[17,20]$. However the synthesis of these molecules is not trivial and large scale production is difficult [17]. Furthermore the peptide sequence variety is until now very limited and each artificial $\beta$-barrel contains only one kind of peptide sequence [21,22].

To overcome the limitations in synthesis and related scale up, the use of $\beta$-barrel proteins modified by genetic engineering techniques can be considered a valid alternative.

Here we report for the first time the successful reengineering of a channel protein with $\beta$-barrel structure leading to an increase in channel diameter. The channel diameter of FhuA variant $\Delta 1-159$ was increased by addition of two $\beta$-strands to the protein primary sequence. As the folding information is intrinsically contained in the primary sequence, it seemed most promising to copy a part of the already existing sequence, therefore the first $\mathrm{N}$-terminal $\beta$-sheet was doubled to reach FhuA variant $\Delta 1-159 \operatorname{Exp}$ (expanded diameter). The FhuA $\Delta 1$ 159 Exp secondary structure was analyzed by circular dichroism (CD). To demonstrate the functionality of FhuA $\Delta$ 1-159 Exp as a channel, kinetics for TMB (3,3',5,5'-tetramethylbenzidine) uptake by HRP (Horse Radish Peroxidase) loaded liposomes with inserted open and biotin label-closed FhuA $\Delta$ 1-159 Exp channel were measured. The kinetic data were compared to results of identically carried out experiments with FhuA $\Delta 1-159$.

\section{Results and Discussion}

\section{FhuA $\Delta$ 1-159 Exp conceptual and estimated increase in} diameter

The aim was to increase the inner channel diameter of $E$. coli outer membrane protein FhuA $\Delta 1-159$, by the addition of a further $\beta$-sheet. A conservative approach was chosen, starting with the addition of only two strands, without introducing entirely new sequence information, but rather by copy-pasting 30 amino acids of the existing sequence. The first two strands on the $\mathrm{N}$-terminus were chosen (Figure 1), as they are

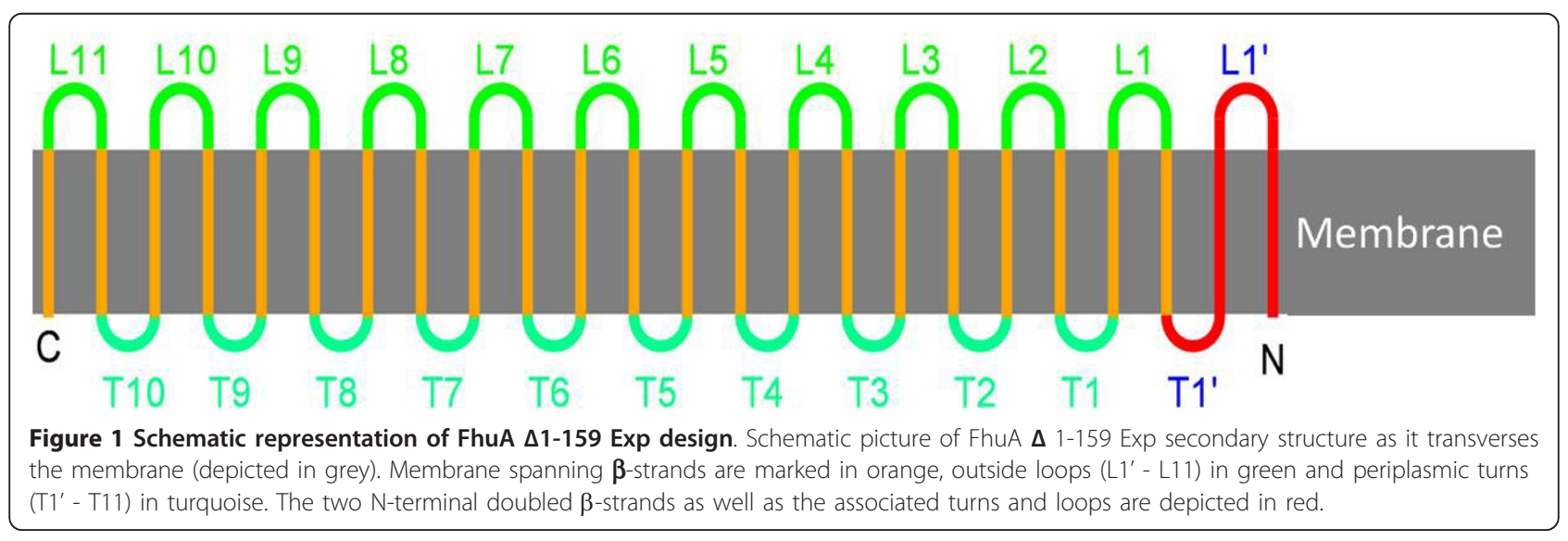


connected by one of the shortest loops. The N-terminal sequence is preserved and thus $\mathrm{N}$ - and $\mathrm{C}$-terminus are still expected to close to the intact barrel structure. Furthermore the chosen $\beta$-stands are rather suited for duplicating, as they are composed $61 \%$ of amino acids that promote $\beta$-sheet formation [23].

Since the FhuA shows a nearly circular morphology $[11,12]$, a simple regular polygonal geometry (hendecagon for FhuA $\Delta 1-159$ and dodecagon for FhuA $\Delta 1-159$ Exp), with constrained side length given by the hydrogen bond connecting the $\beta$-sheets, can be assumed (Figure 2). Based on this assumption the relative expected diameter increase can be calculated, as the FhuA wild type (WT) channel diameter with $22 \beta$-sheets is known to be $\sim 4.2 \mathrm{~nm}$ as deduced from crystal structure $[11,12]$. Knowing the apothem of FhuA WT, the expected FhuA $\Delta 1$-159 Exp pore cross section is $\sim 4.6 \mathrm{~nm}$ as calculated from the apothem ratio. Thus the channel surface area increases by $16 \%$ upon addition of two $\beta$-strands (see also Additional File 1 Table S1).

\section{Extraction and Purification of FhuA $\Delta$ 1-159 Exp}

Detergent extraction led to FhuA $\Delta 1-159$ Exp solubilized in buffer containing detergent oPOE (n-octylpolyoxyethylene). The sample contained many impurities (whole protein content: $\sim 1800 \mu \mathrm{g} / \mathrm{ml}$ ) and was therefore discarded. Second extraction step using the detergent OES (n-octyl-2-hydroxyethylsulfoxide) led to $\sim 420 \mu \mathrm{g} / \mathrm{ml}$ of FhuA $\Delta 1-159$ Exp (Figure 3). ImageJ (http://rsbweb.nih.gov/ij/index.html) analysis resulted in a FhuA $\Delta 1-159$ Exp purity of $\sim 80-90 \%$ for the OES solubilised sample. The ImageJ program converts photographic plates (analogic) to a digitalized matrix where to each pixel ( $\mathrm{x}$ axis) is assigned a value, 0 for white and 1 for black passing through a grey scale (y axis). The total area or number of pixels with values $>$ 0 corresponds to the protein bands on a SDS gel

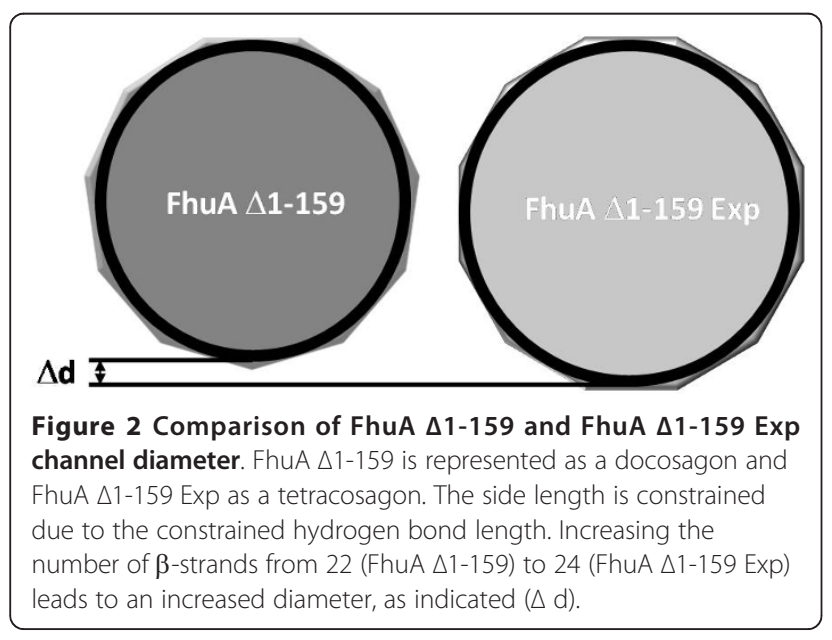

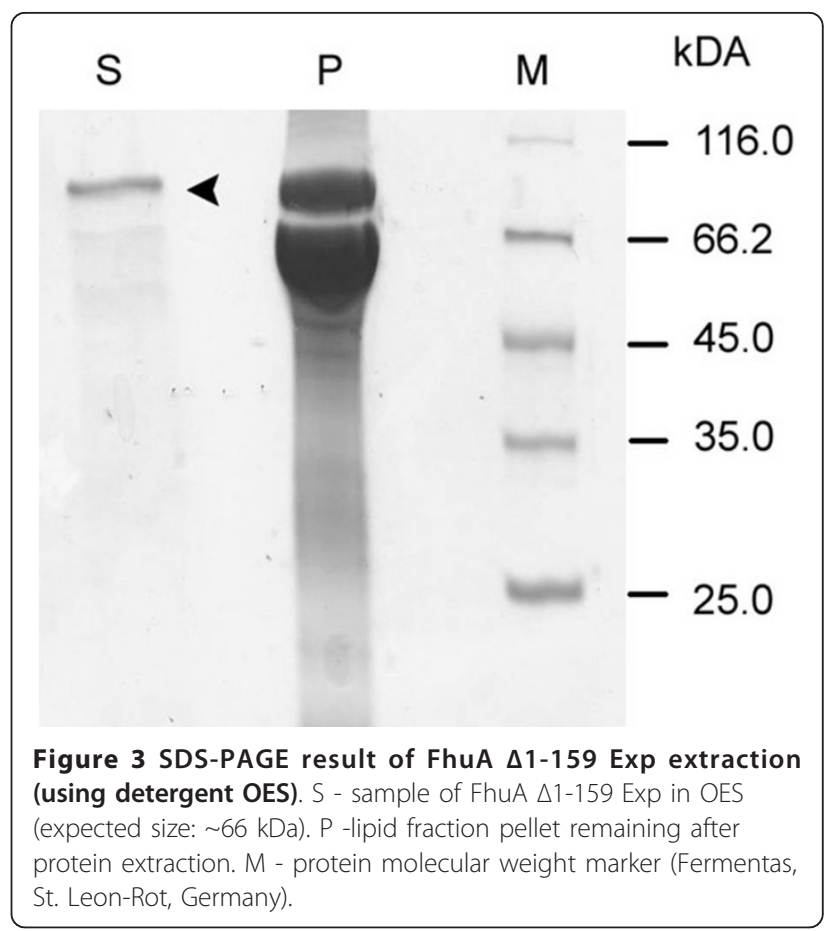

within one lane and is normalized to $100 \%$. At this point the relative purity of a protein in the same lane is simply deduced by determining the ratio between the number of pixels $>0$ corresponding to the interesting protein band and the number of pixels $>0$ of all bands in the lane.

\section{Spectra of FhuA $\Delta$ 1-159 Exp}

Based on the observation that the original FhuA $\Delta 1-159$ is able to independently refold after thermal denaturation (unpublished data from the authors) or when extracted from inclusion bodies [24], showing that folding information is fully contained within the primary sequence [4], the FhuA $\Delta 1-159$ Exp was expected to fold as a $\beta$-barrel.

The secondary structure analysis of FhuA $\Delta 1-159$ Exp by circular dichroism (CD) gives clues on whether the applied engineering strategy to widen the inner protein channel diameter leads still to a $\beta$-sheet folding.

In agreement with previous $C D$ results obtained for WT, FhuA $\Delta 1-159$ as well as WT crystal structure [11], the secondary structure of FhuA $\Delta 1-159$ Exp is well retained as shown in Figure 4.

The CD derived amount of $\beta$-structure for FhuA WT is $51 \%$ [14], while for FhuA $\Delta 1-159$ values between $49 \%$ and $65 \%$ have been reported [24,25].

The deconvolved dichroic spectra using the CONTIN [26] method report a $63 \%$ of $\beta$-strand, $30 \%$ random coil and $7 \% \alpha$-helical contribution for the FhuA $\Delta 1-159 \operatorname{Exp}$ (data fitting and errors are shown in Additional file 2) 


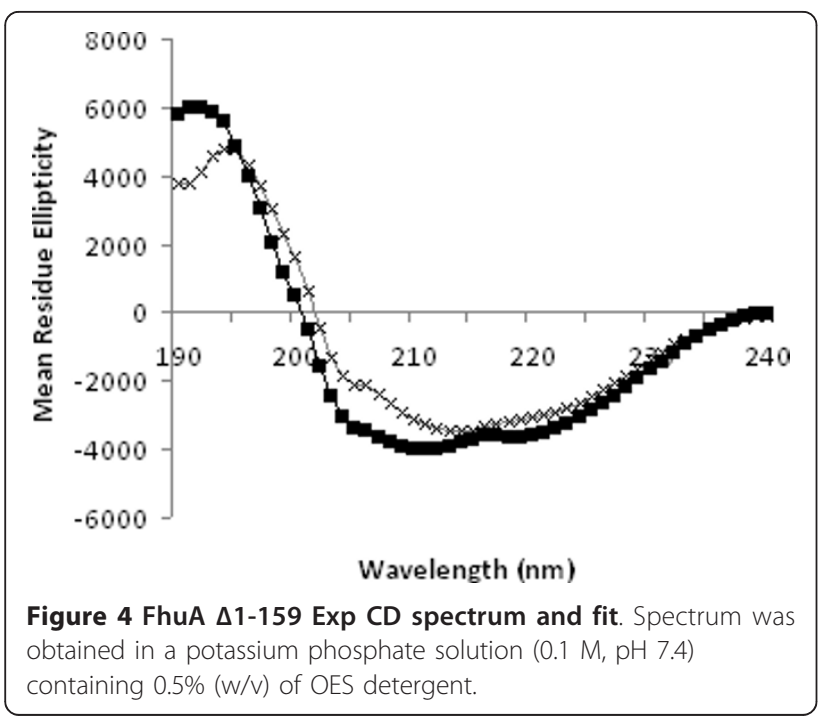

and $57 \%$ of $\beta$-strand, $31 \%$ random coil and $12 \% \alpha$-helical contribution for FhuA $\Delta$ 1-159 (Figure 4) [24].

Though the secondary structure shows the expected trend, the $\mathrm{CD}$ technique cannot provide information on a protein tertiary structure thus the $\beta$-barrel closing cannot definitely be proved. In the following section kinetic studies on liposome inserted FhuA $\Delta$ 1-159 Exp address the channel functionality and suggest a $\beta$-barrel folding.

\section{Influx kinetics and TMB/HRP detection system}

To verify whether FhuA $\Delta 1-159$ Exp is functional, the protein was reconstituted into liposomes made of E. coli lipid extract. The channel functionality was analysed with the help of the HRP/TMB assay system. This method was already applied in order to study the channel properties of membrane isolated FhuA $\Delta 1-159$ [15].

The TMB/HRP is a widely used enzymatic assay, its detection system is based on a two-step irreversible consecutive reaction $\mathrm{A} \rightarrow \mathrm{B} \rightarrow \mathrm{C}(\mathrm{A}=\mathrm{TMB} ; \mathrm{B}$ and $\mathrm{C}=$ first and second TMB oxidation products) catalysed by enzyme HRP in presence of $\mathrm{H}_{2} \mathrm{O}_{2}$. Since the final TMB oxidation product $\mathrm{C}$ is only stable under very acidic conditions $\left(0.3 \mathrm{Mol} / \mathrm{L} \mathrm{H}_{2} \mathrm{SO}_{4}\right)$ [27], the intermediate product (B) is used as a reporter with a characteristic absorbance maximum at 370 and $652 \mathrm{~nm}$. TMB oxidation kinetics were quantified by measuring absorption at $652 \mathrm{~nm}$ over a time of $9 \mathrm{~min}$.

The HRP was encapsulated into liposomes and despite of using the Soret absorption band, the total amount of encapsulated enzyme could not be detected.

The kinetic data obtained in presence of the FhuA $\Delta 1$ 159 Exp, were compared to a negative control consisting of HRP loaded liposomes, to verify the obtained results. Empty liposomes were used as a blank as they show no kinetics and their self-absorption was subtracted from all kinetic data.

Overall kinetic data are reported in Figure 5.

Since the lipid membane is not entirely impermeable, liposomes lacking the channel protein show slow TMB conversion kinetics (Figure 4, squares), however TMB conversion of lipid vesicles with inserted FhuA $\Delta 1-159$ Exp (Figure 4, crosses) occurs 11-times faster. A linear regression using "least square" method was performed to find the best linear section in the steepest region of both curves, to determine the absorbance time derivative (see details in Additional File 1, Figure S2). Lambert Beer Law was used to calculate the TMB conversion speed in $\mathrm{nM} / \mathrm{sec}$ (Table 1) [24]. To address the question whether the FhuA $\Delta 1-159$ Exp truly acts as a channel or whether the liposome membrane gets locally perturbed by the presence of the protein, FhuA $\Delta 1-159$ Exp was blocked by biotinylation of the $31 \mathrm{Lys}-\mathrm{NH}_{2}$ groups, using a technique that has already been applied to block the FhuA $\Delta 1-159[14,15]$ and FhuA $\Delta 1-159$ Ext [16] (see next paragraph).

The previous experiments showed the ability of channel Lys- $\mathrm{NH}_{2}$ group biotinylation to efficiently close the channel in case of FhuA $\Delta 1-159$ (means no detectable substrate conversion) or to at least a partial closing of the channel in case of the elongated FhuA $\Delta 1-159$ Ext (means drastical decrease of substrate conversion speed).

In comparison to liposomes harbouring the open FhuA $\Delta$ 1-159 Exp, liposomes with the blocked channel protein show a $\sim 3$ times smaller slope and TMB conversion rate (Figure 4, black triangles) (see Additional File 1, Figure S2). The incomplete channel blocking can be most likely explained by the expected increase in channel diameter. As the two extra Lys- $\mathrm{NH}_{2}$ groups that were introduced by the addition of two $\beta$-strands should face the channel exterior (as in the original $\beta$-strands in FhuA $\Delta 1-159)$, labelling of these two functional groups

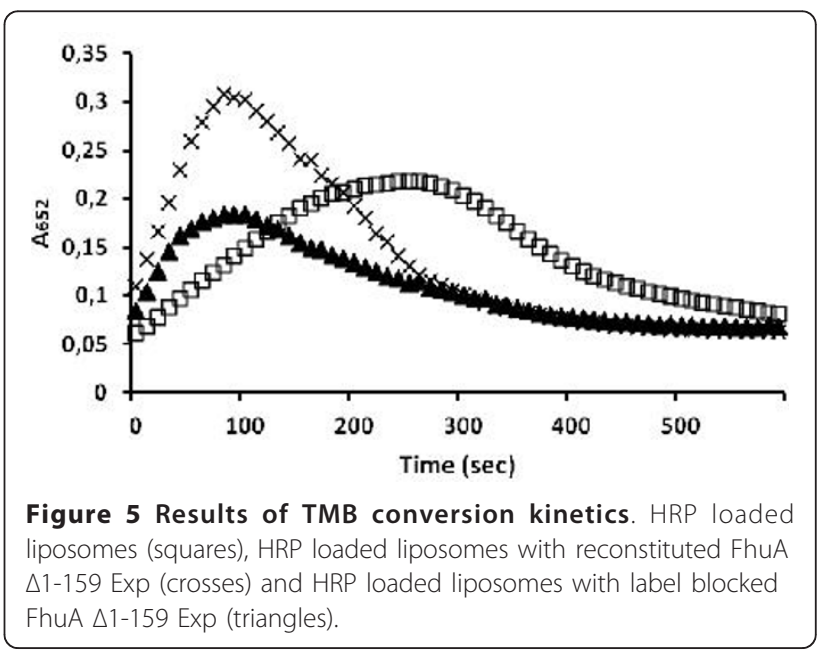


Table 1 Comparison of FhuA $\Delta$ 1-159 and FhuA $\Delta$ 1-159 Exp (unblocked and blocked) average TMB conversions in liposomes

\begin{tabular}{lll}
\hline Sample & $\begin{array}{l}\text { TMB conversion } \\
\text { [nM]/sec }\end{array}$ & Study \\
\hline $\begin{array}{l}\text { Liposomes + HRP + FhuA } \triangle 1-159 \\
\text { Exp }\end{array}$ & $162 \pm 3$ & This study \\
\hline $\begin{array}{l}\text { Liposomes + HRP + FhuA } \Delta 1-159 \\
\text { Exp (blocked) }\end{array}$ & $54 \pm 2$ & This study \\
\hline Liposomes + HRP + FhuA $\triangle 1-159$ & $139 \pm 7$ & {$[24]$} \\
\hline $\begin{array}{l}\text { Liposomes + HRP + FhuA } \triangle 1-159 \\
\text { (blocked) }\end{array}$ & $14 \pm 1$ & {$[24]$} \\
\hline
\end{tabular}

can therefore give no contribution to the blocking. The possibility that not all 31 Lys- $\mathrm{NH}_{2}$ groups were labelled could be excluded by determining the overall amount of biotin labels present on the protein (as described in the next section: "Quantitative determination of the biotinylated Lys") and comparing to expected theoretical amount of labels.

A comparison of FhuA $\Delta$ 1-159 Exp kinetic data and data obtained for FhuA $\Delta 1-159$ as derived from a previous study [24] is shown in Table 1 . It shows that TMB conversion and therefore TMB translocation through the liposomes inserted FhuA $\Delta 1-159$ Exp occurs $\sim 1.2$-times (17\%) faster as compared to FhuA $\Delta 1-159$, this can be correlated to the expected channel surface area increase of 16\% (see Figure 2 and Additional File 1, Table S1).

In fact due to the first Fick's law, the total flux is proportional to the diffusion coefficient and the concentration gradient. As both variables were controlled by the experimental conditions maintaining the same values via the substrate concentration, the ratio (17\%) between the two fluxes measured for the FhuA $\Delta 1-159$ and FhuA $\Delta 1-159$ Exp are only proportional to the surface areas of the two channel with different diameters (16\% higher for FhuA $\Delta$ 1-159 Exp).

TMB conversion through the biotinylated FhuA $\Delta 1$ 159 Exp takes place $\sim 3.7$-times faster than in case of the labelled FhuA $\Delta 1-159$, showing ones more that the increase in inner channel diameter leads to less efficient closing of FhuA $\Delta$ 1-159 Exp.

The observed increase in diffusion kinetics through FhuA $\Delta 1-159$ Exp as compared to FhuA $\Delta 1-159$ strongly suggest that the addition of the two $\beta$-strands led to a diameter increase thus implying the correct folding of added $\beta$-strands.

This conclusion is based on the assumption that the number of channel proteins per liposome (FhuA $\Delta 1-159$ and FhuA $\Delta 1-159$ Exp) are equal. Unfortunately we have not been able to demonstrate this equality in the number.

Therefore the good correlation between channel surface area increase and kinetic results can be either due to an increase in channel diameter or to an unlikely increase in the number of inserted protein channels of exactly $16 \%$.

\section{Quantitative determination of the biotinylated Lys (biotinylation assay)}

Determination of the number of effectively labelled Lys$\mathrm{NH}_{2}$ groups present in the FhuA $\Delta$ 1-159 Exp gives a clue on whether the differences in FhuA $\Delta 1-159$ Exp influx kinetics are due to insufficient labelling or rather and as expected to the diameter increase. FhuA $\Delta 1-159$ Exp contains 31 Lys residues in total. An average biotin concentration of $\sim 4000$ pmol was found after proteolytic digestion of the labelled FhuA $\Delta 1-159$ Exp (exposing all biotin moieties), matching the theoretical biotin label concentration of $4185 \mathrm{pmol}$ in case all 31 Lys are labelled (see paragraph "Biotinylation Assay" in Additional File 1). Therefore the residual kinetics cannot be accounted to a partial FhuA $\Delta$ 1-159 Exp labelling.

\section{Conclusions}

Recently many efforts have been devoted to obtain synthetic pores with $\beta$-barrel conformation having vast technological application ranging from drug-release, host sensors and catalysis [18]. These artificial $\beta$-barrels can in principle be tailored in size and functional groups $[17,20]$. An alternative strategy involves the engineering of bacterial $\beta$-barrel proteins that can be altered in the geometry (diameter, length) and functional groups. A first step in the direction of biological $\beta$-barrels with tailored geometry is the previously reported increase of the FhuA $\Delta 1-159$ length by $1 \mathrm{~nm}$ [16].

In this study in order to complete the channel geometry engineering, the FhuA $\Delta 1-159$ area was increased by $16 \%$ by increasing the number of $\beta$-strands from 22 to 24. To our knowledge this is the first time, besides our previous study [16], a channel protein was specifically engineered to modify its geometry.

A simple "copy-paste" strategy applied to the first two $\beta$-strands at the FhuA $\Delta 1-159 \mathrm{~N}$-terminus has been developed, resulting in protein variant FhuA $\Delta 1-159$ Exp (Expanded). In order to minimize the probability of incorrect protein folding a conservative approach has been followed by adding only two $\beta$-strands. The pasted amino acids are expected to lead to the same folding as the original ones, as the folding information is fully contained in the primary sequence [4]. Considering the cross section of FhuA $\Delta 1-159$ Exp as circular, the channel diameter was increased by $0.4 \mathrm{~nm}$.

FhuA $\Delta 1-159$ Exp was functionally embedded into liposome membranes as confirmed by HRP/TMB assay kinetic studies. Furthermore the kinetic studies revealed an increase in diffusion kinetics of $17 \%$ for FhuA $\Delta 1$ 159 Exp as compared to data obtained for FhuA $\Delta 1$ - 
159 , well in correlation with the $16 \%$ increase in total channel surface area.

The secondary structure analysis by CD spectroscopy suggests the correct $\beta$-barrel folding of the engineered FhuA $\Delta 1-159$ Exp. Overall results suggest that massive FhuA $\Delta$ 1-159 engineering (addition of two $\beta$-strands) is possible without losing channel functionality.

In the future, a combination of the developed FhuA variants with increased channel length and/or diameter will give rise to a new set of synthetic channels with flexible geometry.

\section{Methods}

All chemicals were of analytical grade or higher and purchased from Applichem (Darmstadt, Germany) or Sigma-Aldrich (St. Louis, USA). Protein concentrations were determined using the standard BCA kit (Pierce Chemical Co, Rockford, USA). The oPOE detergent was obtained from Enzo (Farmingdale, USA), while detergent OES was obtained from Bachem (Bubendorf, Switzerland).

\section{Construction and cloning of FhuA $\Delta$ 1-159 Expanded (FhuA $\Delta$ 1-159 Exp)}

To increase the diameter of the FhuA $\Delta 1-159$ the first two $\beta$-sheets ( 30 additional amino acids in total) of the protein were copied and pasted to the $\mathrm{N}$-terminus of the protein (see Figure 1).

After gene design and codon optimisation to $E$. coli using the Gene Designer software (DNA2.0 2007), the synthetic gene "fhuA $\Delta 1-159$ expanded" (fhuA $\Delta$ 1-159 exp) was purchased from Mr. Gene (Regensburg, Germany). The synthetic gene (see paragraph "DNA sequence of FhuA $\Delta$ 1-159 Exp", Additional File 1) was subcloned into the $E$. coli expression vector pBR-IBA1 (IBA, Göttingen, Germany) resulting in plasmid pBRIBA1 fhuA $\Delta 1-159$ exp.

\section{Expression and Extraction of FhuA $\Delta$ 1-159 Exp}

FhuA $\Delta$ 1-159 Exp was expressed in E. coli BE strain BL 21 (DE3) omp8 (F- hsdSB (rB- mB) gal ompT dcm (DE3) $\Delta$ lamB ompF::Tn5 $\Delta$ ompA $\Delta$ ompC) [29] as described previously [7] with some minor changes. Several colonies of freshly transformed E. coli omp8 cells were inoculated into $20 \mathrm{ml} \mathrm{NaPY}_{\text {Amp }}$ medium [30]. This pre-culture was grown over night at $37^{\circ} \mathrm{C}$. The main-culture was inoculated from the pre-culture to an initial $\mathrm{OD}_{600}$ of 0.2 . Expression was performed in $200 \mathrm{ml} \mathrm{NaPY} \mathrm{Amp}_{\mathrm{mp}}$ medium at $30^{\circ} \mathrm{C}$. Protein expression was induced by adding $1 \mathrm{mM}$ IPTG at an $\mathrm{OD}_{600}$ of 0.7-0.8. The expression level of FhuA $\Delta 1-159$ Exp was monitored by SDS PAGE (see section SDS PAGE). After reaching stationary phase cells were harvested by centrifugation at $3.300 \times \mathrm{g}, 4^{\circ} \mathrm{C}, 15 \mathrm{~min}$ and the resulting pellet was stored at $-20^{\circ} \mathrm{C}$ until protein extraction.

FhuA $\Delta 1-159$ Exp was extracted from the E. coli outer membrane as previously described [31] with slight changes. All amounts are given for a pellet obtained from a $200 \mathrm{ml}$ cell culture with an $\mathrm{OD}_{600}$ of 3.0.

The cell pellet was resuspended in $10 \mathrm{ml}$ lysis buffer (0.1 $\mathrm{M}$ phosphate-buffer, $\mathrm{pH} 7.4 ; 2.5 \mathrm{mM} \mathrm{MgCl}_{2} ; 0.1$ $\mathrm{mM} \mathrm{CaCl}$ ). By the use of a high pressure homogenizator (EmulsiFlex-C3, Avestin, Ottawa, Canada) the cells were broken. Thereafter $1 \mathrm{ml}$ of extraction buffer $(0.1$ $\mathrm{M}$ phosphate-buffer, pH 7.4; $2.5 \mathrm{mM} \mathrm{MgCl}_{2} ; 0.1 \mathrm{mM}$ $\mathrm{CaCl}_{2} ; 20 \% \mathrm{v} / \mathrm{v}$ Triton $\left.\mathrm{X}-100\right)$ was added and samples were incubated while shaking at $37^{\circ} \mathrm{C}$ for 1 hour. After incubation the solution was centrifuged at $38000 \times \mathrm{g}, 4^{\circ}$ $\mathrm{C}$ for $45 \mathrm{~min}$. Supernatant was discarded and the pellet was washed 3 times with washing buffer $(0.1 \mathrm{M}$ phosphate-buffer, $\mathrm{pH}$ 7.4) without resuspending it. The remaining pellet was homogenized in $1.5 \mathrm{ml}$ pre-extraction buffer $(0.1 \mathrm{M}$ phosphate-buffer, $\mathrm{pH} 7.4 ; 1 \mathrm{mM}$ EDTA; $0.1 \% \mathrm{v} / \mathrm{v}$ oPOE) using a tissue grind tube. Afterwards the solution was incubated at $37^{\circ} \mathrm{C}$ for 1 hour and the next centrifugation was carried out at 170000 $\mathrm{xg}, 4^{\circ} \mathrm{C}$ for $45 \mathrm{~min}$ (Beckmann Coulter Optima ${ }^{\mathrm{TM}}$, L100-XP Ultracentrifuge, California USA). Again the pellet was resuspended using the tissue grind tube in 1.5 $\mathrm{ml}$ solubilisation buffer $(0.1 \mathrm{M}$ phosphate-buffer, $\mathrm{pH} 7.4$; $1 \mathrm{mM}$ EDTA; $3.0 \% \mathrm{v} / \mathrm{v}$ oPOE) and incubated for 1 hour at $37^{\circ} \mathrm{C}$. The last centrifugation step was performed at $170000 \mathrm{xg}, 12^{\circ} \mathrm{C}$ for $45 \mathrm{~min}$ (Beckmann Coulter Optima $^{\mathrm{TM}}, \mathrm{L}-100-\mathrm{XP}$ Ultracentrifuge, California USA) to obtain the FhuA $\Delta$ 1-159 Exp protein in the supernatant. At all steps $1 \mu$ l phenylmethanesulfonyl fluorid (PMSF; protease inhibitor) was added.

From each pellet and supernatant samples were taken and analyzed on SDS gel.

To increase the FhuA $\Delta 1-159$ Exp yield, the residual pellet from the oPOE-extraction was used for further extraction with the detergent OES [32]. Therefore the pellet was resuspended in $1.5 \mathrm{ml} \mathrm{OES}$-solubilisation buffer (0.1 M phosphate-buffer, pH 7.4; $1 \mathrm{mM}$ EDTA; 0.5\% $\mathrm{w} / \mathrm{v}$ OES) using the tissue grind tube. After proper resuspension the solution was incubated 1 hour at $37^{\circ} \mathrm{C}$ with shaking. Subsequently it was centrifuged at 170000 $\mathrm{xg}, 12^{\circ} \mathrm{C}$ for $45 \mathrm{~min}$ to obtain the FhuA $\Delta 1-159 \operatorname{Exp}$ protein in the supernatant.

The purified FhuA $\Delta 1-159$ Exp was loaded onto a $10 \%$ SDS acrylamide gel [33]. After electrophoresis the protein was stained by Coomassie Brilliant blue R-250.

\section{SDS-PAGE}

SDS polyacrylamide gel electrophoresis utilizing gels consisting of a $10 \% \mathrm{w} / \mathrm{v}$ acrylamide separation gel with a $5 \% \mathrm{w} / \mathrm{v}$ acrylamide stacking gel were used to monitor 
the protein expressions as well as to evaluate the quality of protein purification. Bands were visualized by Coomassie staining [33].

\section{Secondary structure determination of FhuA $\Delta$ 1-159 Exp by circular dichroism (CD) spectroscopy}

Dichroic spectra were measured with an Olis DSM 17 CD spectrophotometer (Olis, Bogard, USA). Samples were pipetted into a Suprasil QS cuvette (Hellma, Müllheim, Germany) with a pathlenght of $0.5 \mathrm{~nm}$. For each samples five spectra from $190 \mathrm{~nm}$ to $240 \mathrm{~nm}$ were collected and averaged. Background spectra using the buffer, in which the protein was solved, were subtracted.

Spectra were analyzed to calculate the $\alpha$-helix, $\beta$-barrel and random coil secondary structure content by using the Dicroprot deconvolution program [34] and the CONTIN algorithm [35].

\section{Liposome preparation}

Liposomes were formed using the film hydration method [15] followed by a filter extrusion process and finally a size exclusion chromatography (SEC) for homogenization and purification.

For the lipid film preparation $500 \mu \mathrm{l}$ of E. coli total lipid extract $(10 \mathrm{mg})$ dissolved in chloroform (Avanti Polar Lipids, Alabasta, USA) were mixed 1:1 with methanol. The organic solvents were evaporated in a round bottom flask using a vacuum evaporator (VWR, Darmstadt, Germany) to create a lipid film in the flask. HRP (horse radish peroxidase) loaded liposomes were obtained by adding $1 \mathrm{ml}$ of an aqueous buffer solution (0.1 M potassium phosphate-buffer, $\mathrm{pH}$ 7.4) containing HRP $(2.9 \mathrm{U} / \mathrm{ml})$ to the lipid film. Liposomes with entrapped HRP and membrane inserted FhuA $\Delta$ 1-159 $\operatorname{Exp}(2.7 \mu \mathrm{M})$ or FhuA $\Delta 1-159(2.7 \mu \mathrm{M})$ respectively (as well as Lys- $\mathrm{NH}_{2}$ labeled forms of both channel proteins) were prepared by adding $1 \mathrm{ml}$ buffer $(0.1 \mathrm{M}$ potassium phosphate-buffer, $\mathrm{pH}$ 7.4) containing HRP $(2.9 \mathrm{U} / \mathrm{ml})$ as well as the protein to insert $(2.7 \mu \mathrm{M})$ to the previously formed lipid film. Empty liposomes as they were used as negative controls, were rehydrated by the addition of $1 \mathrm{ml}$ buffer $(0.1 \mathrm{M}$ potassium phosphate-buffer, $\mathrm{pH}$ 7.4). Liposomes were then formed via rehydration, under slow rotation of the round bottom flasks. Lipid film preparation and rehydration were carried out over night.

Rehydrated liposomes were sequentially extruded with an Avanti polar lipids extruder (Avanti Polar Lipids, Alabasta, USA) with $1 \mu \mathrm{m}, 0.4 \mu \mathrm{m}$ and $0.2 \mu \mathrm{m}$ membranes (Millipore, Bedford, USA) to uniform the nanocompartments in size and shape [36]. Purification was carried out through size exclusion chromatography (SEC).

\section{Size exclusion chromatography (SEC) for liposome purification}

All five subsets of produced nanocompartments (liposomes without inserted channel protein, liposomes with inserted FhuA $\Delta 1-159 \mathrm{Exp}$ as well as liposomes with inserted FhuA $\Delta 1-159$ and the labelled forms of both channel proteins) were purified by gel filtration using Sepharose 6B (Sigma-Aldrich, Cat. no. 6B100-500 ML) in phosphate buffer $(0.1 \mathrm{M}$ potassium phosphate-buffer, $\mathrm{pH}$ 7.4).

First the SEC column was saturated with liposomes to avoid unspecific binding to the matrix. Afterwards liposomes obtained from the extrusion process were loaded. Fractions of $750 \mu \mathrm{l}$ were collected and Average diameters of nanocompartments were routinely determined using a Zeta-Sizer (Zeta-Sizer Nano Series; Malvern, Worcestershire, UK//Supp. Mat.: Additional File 1, Figure S1).

\section{TMB assay with liposomes}

As a reporter system for compound influx into the liposomes a colorimetric TMB conversion assay was used. Since in all types of prepared liposomes HRP is encapsulated in the lumen TMB gets converted in a two-step oxidation reaction into a blue $(E=650 \mathrm{~nm})$ and subsequently yellow $(E=420 \mathrm{~nm})$ product after entering the liposome.

To $50 \mu \mathrm{l}$ of liposome solution (normalized to a $\mathrm{OD}_{600}$ of $0.04 / \mathrm{ml})$ and $50 \mu \mathrm{l}$ of phosphate buffer $(0.1 \mathrm{M}$ phosphate-buffer, $\mathrm{pH}$ 7.4) $20 \mu \mathrm{l}$ of TMB-substrate solution (readymade $\mathrm{TMB} / \mathrm{H}_{2} \mathrm{O}_{2}$ solution; Sigma-Aldrich, St. Louis, USA) were added. TMB oxidation kinetics was monitored at $650 \mathrm{~nm}$ in a Tecan Infinate M1000 spectrofluorometer (Tecan, Männedorf, Switzerland).

\section{FhuA $\Delta$ 1-159 Exp and FhuA $\Delta$ 1-159 labelling}

In order to biotinylate the Lys- $\mathrm{NH}_{2}$ groups present on FhuA $\Delta 1-159$ Exp and FhuA $\Delta 1-159$, a 20\% DMSO aqueous solution containing (2-[Biotinamido] ethylamido)3,3'-dithiodipropionic acid $N$-hydroxysuccinimide ester) $(8.2 \mathrm{mM})$ was added drop-wise to $1200 \mu \mathrm{l}$ of a FhuA $\Delta$ 1-159 Exp or FhuA $\Delta 1-159$ solution respectively and stirred (3000 rpm, $1 \mathrm{~h}$; RCT basic IKAMAG, IKAWerke $\mathrm{GmbH}$, Staufen, Germany). The latter solution was used for the formation of liposomes, as described above as well as for the biotinylation assay.

\section{Quantitative determination of the biotinylated Lys (biotinylation assay)}

The determination of the biotinyl groups present on the FhuA $\Delta$ 1-159 Exp and FhuA $\Delta 1-159$ protein has been performed using the Invitrogen FluoReporter ${ }^{\circledR}$ Biotin Quantitation Assay Kit specifically developed for proteins. Fluorescence was detected by a Tecan 
Spectrofluorometer (Infinite ${ }^{\circledR}$ 1000, Tecan Group Ltd., Männedorf Switzerland).

\section{Additional material}

Additional file 1: • Liposome DLS Data. - FhuA $\triangle 1$ 159 Exp estimated increase in diameter $\cdot$ HRP Assay $\cdot$ Biotinylation Assay $\cdot$ DNA sequence of FhuA $\triangle 1-159$ Exp.

Additional file 2: • CD data and CONTIN deconvolution output for FhuA $\Delta$ 1-159 Exp.

\section{Acknowledgements}

MK acknowledge Prof. Ulrich Schwaneberg and RWTH Aachen University for the possibility to develop this work.

\section{Authors' contributions}

MK and TD carried out design and performed study, data analysis and drafting of the manuscript.

MF designed research.

All author's read and approved the final manuscript.

\section{Competing interests}

The authors declare that they have no competing interests.

Received: 14 July 2011 Accepted: 19 August 2011

Published: 19 August 2011

\section{References}

1. Schulz G: Porins: general to specific, native to engineered passive pores. Curr Opin Struct Biol 1996, 6:485-490.

2. Kleinschmidt $J H, K T$ T: Secondary and tertiary structure formation of the b-barrel membrane protein OmpA is synchronized and depends on membrane thickness. J Mol Biol 2002, 324:319-330.

3. Ramachandran R, Heuck AP, Tweten RK, Johnson AE: Structural insights into the membrane-anchoring mechanism of a cholesterol-dependent cytolysin. Nat Struct Biol 2002, 9:823-827.

4. Wimley C: The versatile beta-barrel membrane protein. Curr Opin StruC Biol 2003, 13:404-411.

5. Ye J, van den Berg B: Crystal structure of the bacterial nucleoside transporter Tsx. EMBO J 2004, 23:3187-3195.

6. Cowan SW, Garavito RM, Jansonius JN, Jenkins JA, Karlsson R, Konig N, Pai EF, Pauptit RA, Rizkallah PJ, Rosenbach JP: The structure of OmpF porin in a tetragonal crystal form. Structure 1995, 3:1041-1050.

7. Nallani M, Benito S, Onaca O, Graff A, Lindemann M, Winterhalter M, Meier W, Schwaneberg U: A nanocompartment system (synthosome) designed for biotechnological applications. J Biotechnol 2006, 123:50-59.

8. Ranquin A, Versees W, Meier W, Steyaert J, Van Gelder P: Therapeutic nanoreactors: combining chemistry and biology in a novel triblock copolymer drug delivery system. Nano Lett 2005, 5:2220-2224.

9. Surrey T, Schmid A, Jähnig F: Folding and membrane insertion of the trimeric beta-barrel protein OmpF. Biochemistry 1996, 35:2283-2288.

10. Hoffmann $H$, Fischer $E$, Schwarz $H$, Braun V: Overproduction of the proFhuA outer membrane receptor protein of Escherichia coli K-12: isolation, properties, and immunocytochemical localization at the inner side of the cytoplasmic membrane. Arch Microbiol 1986, 145:334-341.

11. Ferguson A, Braun V, Fiedler H-P, Coulton J, Diederichs K, Welte W: Crystal structure of the antibiotic albomycin in complex with the outer membrane transporter FhuA. Prot Sci 2000, 9:956-963.

12. Ferguson A, Hofmann E, Coulton J, Diederichs K: Siderophore-mediated iron transport: crystal structure bound lipopolysaccharide. Science 1998, 282:2215-2220.

13. Braun M, Killmann $H$, Maier $E$, Benz $R$, Braun V: Diffusion through channel derivatives of the Escherichia coli FhuA transport protein. Eur J Biochem 2002, 269:4948-4959.

14. Onaca O, Sarkar P, Roccatano D, Friedrich T, Hauer B, Grzelakowski M, Güven A, Fioroni M, Schwaneberg U: Functionalized nanocompartments
(Synthosomes) with a reduction-triggered release system. Ang Chem Int Ed 2008, 47:7029-7031.

15. Güven A, Fioroni M, Hauer B, Schwaneberg U: Molecular understanding of sterically controlled compound release through an engineered channel protein (FhuA). J Nanobiotech 2010, 8(14).

16. Muhammad N, Dworeck T, Fioroni M, Schwaneberg U: Engineering of the E. coli Outer Membrane Protein FhuA to overcome the Hydrophobic Mismatch in Thick Polymeric Membranes. J Nanobiotech 2011, 9(8).

17. Baumeister B, Matile S: Rigid-rod beta-barrels as lipocalin models: probing confined space by carotenoid encapsulation. Chem - Eur J 2000, 6:1739-1749.

18. Sakai N, Matile S: Synthetic multifunctional pores: lessons from rigid-rod beta-barrels. Chem Commun 2003, 2514-2523.

19. Schwab PFH, Levin MD, Michl J: Molecular rods. 1. Simple axial rods. Chem Rev 1999, 99:1863-1934.

20. Litvinchuk S, Bollot G, Mareda J, Som A, Ronan D, Shah MR, Perrottet P, Sakai N, Matile $S$ : Thermodynamic and kinetic stability of synthetic multifunctional rigid-rod $\beta$-barrel pores: evidence for supramolecular catalysis. J Am Chem Soc 2004, 126:10067-10075

21. Kumaki J, Yashima E, Bollot G, Mareda J, Litvinchuk S, Matile S: AFM snapshots of synthetic multifunctional pores with polyacetylene blockers: Pseudorotaxanes and template effects. Angew Chem, Int Ed 2005, 44:6154-6157.

22. Litvinchuk S, Matile S: Blockage of rigid-rod beta-barrel pores by rigid-rod R-helix mimics. Supramol Chem 2005, 17:135-139.

23. Cantor CR, Schimmel PR: The conformation of biological macromoleculs New York: W. H. Freeman and Company; 1980.

24. Dworeck T, Petri AK, Muhammad N, Fioroni M, Schwaneberg U: FhuA Deletion Variant del1-159 Overexpression in Inclusion Bodies and Refolding with Polyethylene-Poly(ethylene glycol) Diblock Copolymer. Prot Expr Purif 2011, 77(1):75-79.

25. Boulanger $P$, le Marie $M$, Bonhivers $M$, Dubois S, Desmadril S, Letellier L: Purification and structural and functional characterization of FhuA, a transporter of the Escherichia coli outer membrane. Biochemistry 1996 35:14216-14224

26. Andrade M, Chacón P, Merelo J, Morán F: Evaluation of secondary structure of proteins from UV circular dichroism using an unsupervised learning neural network. Prot Eng 1993, 6:383-390.

27. Josephy P, Eling T, Mason R: The horseradish peroxidase-catalyzed oxidation of $3,5,3^{\prime}, 5^{\prime}$-tetramethylbenzidine. Free radical and chargetransfer complex intermediates. J of Biol Chem 1982, 257:3669-3675.

28. Josephy $P$, Eling T, Mason R: The horseradish peroxidase-catalyzed oxidation of $3,5,3^{\prime}, 5^{\prime}$-tetramethylbenzidine. Free radical and chargetransfer complex intermediates. J Biol Chem 1982, 257:3669-3675.

29. Prilipov A, Phale P, Koebnik R, Widmer C, Rosenbusch J: Identification and characterization of two quiescent porin genes, $\mathrm{nmpC}$ and ompN, in Escherichia coli B. J Bacteriol 1998, 180:3388-3392.

30. Onoda T, Enokizono J, Kaya H, Oshima A, Freestone P, Norris V: Effects of calcium and calcium chelators on growth and morphology of Escherichia coli L-form NC-7. J Bacterio/ 2000, 182(5):1419-1422.

31. Locher KP, Rosenbusch JP: Oligomeric states and siderophore binding of the ligand-gated FhuA protein that forms channels across Escherichia coli outer membranes. Eur J Biochem 1997, 247(3):770-775.

32. Locher KP, Rees B, Koebnik R, Mitschler A, Moulinier L, Rosenbusch JP, Moras D: Transmembrane signaling across the ligand-gated FhuA receptor: crystal structures of free and ferrichrome-bound states reveal allosteric changes. Cell 1998, 95:771-778.

33. Laemmli U: Cleavage of structural proteins during the assembly of the head of bacteriophage T4. Nature 1970, 227:680-685.

34. Deléage $\mathrm{G}$, Geourjon C: An interactive graphic program for calculating the secondary structures content of proteins from circular dichroism spectrum. Comput Appl Biosci 1993, 2:197-199.

35. Provencher SW: An eigenfunction expansion method for the analysis of exponential decay curves. Comput Phys Commun 1982, 27:213-227.

36. Broz P, Benito SM, Saw C, Burger P, Heider H, Pfisterer M, Marsch S, Meier W, Hunziker P: Cell targeting by a generic receptor-targeted polymer nanocontainer platform. J Control Release 2005, 102:475-488.

doi:10.1186/1477-3155-9-33

Cite this article as: Krewinkel et al.: Engineering of an E. coli outer membrane protein FhuA with increased channel diameter. Journal of Nanobiotechnology 2011 9:33. 